\title{
Importance of patient's perceptions in developing new surgical techniques
}

\author{
Ahsan Rao • Irfan Ahmed
}

Published online: 26 October 2010

(C) Springer Science+Business Media, LLC 2010

We thank Dr. Cavazzola and coauthors for the comments in their Letter to the Editor, "Who should decide the best minimally invasive approach? Should we listen to our patients?"

Most of the issues raised by Dr. Cavazzola are significant but they stand valid in isolation and did not fall within the context of the questionnaire. His team rightly said that single port surgery and NOTES (Natural Orifice Trans Endoscopic Surgery) are not regular procedures. We have already discussed in our paper that these techniques were new and more data are required to prove their safety. The same information was conveyed to the participants of the survey. Similarly, we agree that single port incision can have potential complications, although it still has to be proven. Hence, we did not provide extensive details on benefits and risks of each procedure; otherwise, it would have made the questionnaire laborious to read and bias toward a particular technique.

We would like to reemphasize that the main idea of the paper was to ascertain people's perception about new surgical techniques in general rather than providing details about variety of minimally invasive procedures. Hence, other uncommon new surgical techniques in Scotland, such as needlescopy, were not included in the questionnaire.

Disclosures Dr. Ahsan Rao and Mr. Irfan Ahmed have no conflicts of interest or financial ties to disclose.

\footnotetext{
A. Rao $(\bowtie) \cdot$ I. Ahmed

Aberdeen Royal Infirmary, Aberdeen, UK

e-mail: a.rao.03@aberdeen.ac.uk; ahsan.rao@nhs.net

I. Ahmed

e-mail: irfanahmed2@nhs.net
} 